\title{
Latency Minimization in Wireless IoT Using Prioritized Channel Access and Data Aggregation
}

\author{
Sabin Bhandari, Shree Krishna Sharma, Xianbin Wang \\ Department of Electrical and Computer Engineering, Western University, London, Ontario, Canada \\ Email: \{sbhanda7, sshar323, xianbin.wang\}@uwo.ca
}

\begin{abstract}
Future Internet of Things (IoT) networks are expected to support a massive number of heterogeneous devices/sensors in diverse applications ranging from eHealthcare to industrial control systems. In highly-dense deployment scenarios such as industrial IoT systems, providing reliable communication links with low-latency becomes challenging due to the involved system delay including data acquisition and processing latencies at the edge-side of IoT networks. In this regard, this paper proposes a priority-based channel access and data aggregation scheme at the Cluster Head $(\mathrm{CH})$ to reduce channel access and queuing delays in a clustered industrial IoT network. First, a prioritized channel access mechanism is developed by assigning different Medium Access Control (MAC) layer attributes to the packets coming from two types of IoT nodes, namely, highpriority and low-priority nodes, based on the application-specific information provided from the cloud-center. Subsequently, a preemptive M/G/1 queuing model is employed by using separate low-priority and high-priority queues before sending aggregated data to the Cloud. Our results show that the proposed prioritybased method significantly improves the system latency and reliability as compared to the non-prioritized scheme.
\end{abstract}

Index Terms - Internet of Things (IoT), Latency minimization, Cloud-center, Data aggregation.

\section{INTRODUCTION}

Internet of Things (IoT) has been emerged as a new paradigm that interconnects various objects and processes for distributed real-time information collection and utilization in several applications [1]. A typical IoT architecture mainly consists of four interconnected sub-systems, including connected intelligent objects/things through a sensor network, routers/gateways at the edge, backbone communication infrastructure, and the clouds [2]. Today's developments in the Wireless Sensor Networks (WSNs), Device-to-Device (D2D), Internet, Machine-to-Machine (M2M), and mobile computing technologies have a significant impact to extend the sensory capabilities of IoT networks [3]. However, due to large-scale and highly-dense nature of many IoT applications, performing timely acquisition and analysis of IoT related data is crucial to support low-latency applications.

Among many potential applications, industrial IoT is considered as a key enabler for industrial automation, intelligent transportation, logistics and control systems [4]. Various application requirements have brought many challenges to design more efficient and reliable industrial IoT networks. The main challenges in industrial IoT networks include low latency, low per node energy consumption, reliability, and secure data transmissions to the application servers [5]. Out of these, IoT network latency has been considered as one of the most critical issues in industrial automation and control sub-systems. The main network parameters that affect the system delay are node density, data rate, and energy per node, processing power, routing protocol and Medium Access Control (MAC) protocol [6]. To deal with the latency issue, an IoT network must be designed to meet the real-time requirements of the aforementioned application scenarios [5].

One of the potential approaches to reduce system delay in dense wireless IoT networks is to devise a suitable MAC protocol, which can effectively regulate the access of limited channel resources. At the MAC layer, several factors such as overhearing, over-emitting, collisions, and control packets overhead affect the overall system delay. These factors are generally related to the radio operating mode, the medium access technique and the service time. In this context, a number of MAC protocols have been proposed based on the IEEE 802.15.4 standard [7] to address the latency issues. A MAC protocol based backoff time decision rule has been presented in [8] for a hierarchical M2M network having different clustered nodes. Besides, a mathematical model has been introduced in [9] for superframe and access latency of the MAC protocol for an industrial IoT environment based on the queuing theory. Furthermore, an extended channel access mechanism namely, Explicit Prioritized Channel Access Protocol (EPCAP) [10] has been studied based on the IEEE 802.15.4 standard. The EPCAP proposed in [10] incorporates different traffic priority levels to handle critical events and utilizes $\mathrm{M} / \mathrm{G} / \mathrm{c}$ based multi-server queuing network system.

The level of network latency can be further reduced by dividing incoming data packets into different queues, and subsequently by employing a suitable data aggregation scheme. The data aggregation process helps to eliminate the data redundancy, to minimize the communication load, and hence to reduce the overall network latency. In this regard, the authors in [11] proposed to employ a data aggregation scheme to reduce the network signaling load. In addition, a tunnel based data aggregation method has been proposed in [12], in which an aggregator merges the M2M data packets, appends with its own packet, and forwards the aggregated data to the gateway/base station. Besides, the authors in [13] proposed a priority based data aggregation scheme for M2M communication over the cellular network to maintain the trade-off between delay requirements and power constraints by using a preemptive M/G/1 queuing model. However, the existing 
works did not consider the joint impact of priority based channel access and prioritized queuing in heterogeneous IoT networks. In addition, the potential benefit of involving the cloud in latency reduction at the IoT edge network has not been considered.

In this paper, a cloud-assisted priority-based channel access and data aggregation scheme is proposed for irregularly deployed sensor nodes to minimize the network latency and to enhance the system reliability of IoT networks. The cloud center is equipped with massive processing power, and storage capabilities [14], however, it does not support low-latency applications $[15,16]$. In the considered framework, the Cluster Head $(\mathrm{CH})$ extends the cloud's functions to the edge of the network by prioritizing and aggregating the incoming data packets, and the cloud-center provides various levels of information such as priority levels and locations of the IoT nodes to the $\mathrm{CH}$. A priority based channel access scheme is employed at the $\mathrm{CH}$ to reduce the channel access latency by assigning different MAC layer attributes to the incoming data packets. Subsequently, the prioritized data packets are sent to the separate queues according to their priority levels and are aggregated before sending to the cloud via a gateway. Finally, the performance of the proposed joint prioritized channel access and data aggregation is analyzed using the preemptive $\mathrm{M} / \mathrm{G} / 1$ queuing model and compared with the conventional non-prioritized scheme.

The rest of this paper is organized as follows. In Section II, the overall system model of a hierarchical IoT network is presented. In Section III, the proposed prioritized channel access and data aggregation scheme is described in detail. In Section IV, the performance of the proposed method is evaluated via simulations. Finally, the paper is concluded in Section V.

\section{System ModeL}

In this paper, we consider an industrial IoT scenario, composed of $N$ number of heterogeneous sensor nodes deployed over an area of $l \times l \mathrm{~m}^{2}$ (i.e., rectangular industrial sub-unit) as shown in Fig. 1. In each industrial unit, we classify the data gathered by $N$ sensors into two classes, i.e., normal data (ND) and event driven (ED) data traffic. The ND packets are regularly generated by low priority $P_{l}$ nodes during some process-related measurements, while the sporadic ED packets are triggered by high priority $P_{h}$ nodes when a physical quantity detected by a sensor crosses its threshold. We assume that each node supports only one type of data, i.e., either ND or ED. Also, $M$ out of $N$ nodes transmit high priority packets, i.e., $P_{h}$ packets and the remaining nodes transmit only low priority packets, i.e., $P_{l}$ packets. In addition, the proposed network topology is considered to be static over the time. We assume that the gateway and the cloud-center are connected via high-speed wireless links with negligible latency and packet loss.

All the deployed sensor nodes are associated with the $\mathrm{CH} /$ aggregator. Also, we consider that the nodes including $\mathrm{CH}$ and the gateway have the child-parent relationships. All the

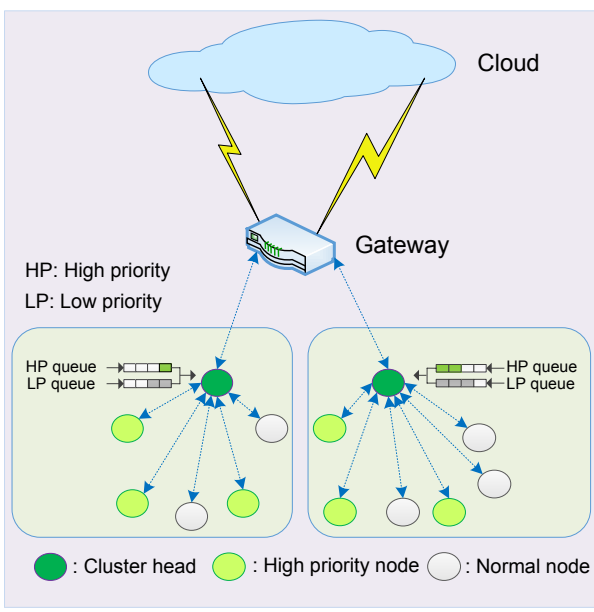

Fig. 1. System model for the hierarchical IoT network.

sensor nodes belonging to the same $\mathrm{CH}$ contend to access the channel to the corresponding parent node of the link. Data generated from terminal nodes are transmitted to the gateway after data aggregation at the $\mathrm{CH}$ for subsequent transmission to the application server. The gateway and $\mathrm{CH}$ are considered to be positioned at the specific locations and usually have the higher energy and computational power as compared to the sensor nodes. The $\mathrm{CH}$ can get the application-specific information such as priority levels and locations from the cloud application server. In the considered system setup, the queuing delay for each priority class depends on the scheduling policy adopted at the $\mathrm{CH}$.

The M/G/1 queuing method can be used to model the stochastic behavior of a device to measure the network performances such as throughput, latency, packet loss probability, and energy consumption [17]. The M/G/1 queuing system with priorities can be further classified into non-preemptive and preemptive queuing models. In the case of non-preemptive priority packet scheduling, when a low priority packet $P_{l}$ starts execution, the current processing task continues even if the higher priority packet $P_{h}$ arrives at the queue. In addition, $P_{h}$ packets have to wait in the queue until the task of $P_{l}$ packets becomes complete. However, in the preemptive priority packet scheduling, the higher priority packets $P_{h}$ are processed first and can preempt the lower priority packets by saving their contexts, if they are already executing the task [13]. In the considered system, we propose to employ the preemptive $\mathrm{M} / \mathrm{G} / 1$ priority queuing model at the $\mathrm{CH}$, as detailed later in Section III.

\section{A. System Delay}

In an IoT network, the overall system latency depends on a set of parameters such as distance and the number of hops towards the destination node, data rate, node density, MAC and routing protocols, and the available energy and computational resources at the nodes. All the above-mentioned parameters may lead to unpredictable and high end-to-end latency. Out of these, the employed MAC layer protocol determines the onehop delay and the network layer is responsible for controlling the multi-hop delay. The one-hop delay $\tau_{h d}$ resulted at the 


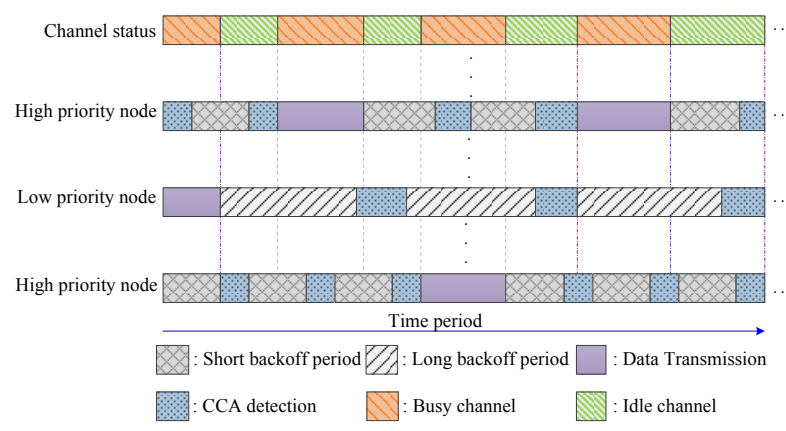

Fig. 2. Prioritized channel access mechanism.

MAC layer can be expressed in terms of different delay components as follows [18]

$$
\tau_{h d}=\tau_{p r d}+\tau_{q d}+\tau_{c d}+\tau_{t d}+\tau_{p g d}+\tau_{r d},
$$

where $\tau_{t d}, \tau_{r d}$, and $\tau_{p g d}$ denote transmission, reception, and propagation delays, respectively, and are hardware dependent. Similarly, $\tau_{p r d}, \tau_{c d}$, and $\tau_{q d}$ are the processing, channel access, and queuing delays, respectively, and higher latency may result due to the queuing of the packets and the time required to access a channel. The critical MAC layer challenge for IoT networks is to facilitate the channel access to an extremely large number of devices with unique traffic characteristic and diverse service requirements. In this regard, this paper focuses on improving queuing strategies and channel access techniques to ensure Quality of Service (QoS) requirements in terms of latency and reliability.

\section{Proposed Prioritized Channel Access AND Data Aggregation Scheme}

\section{A. Prioritized Channel Access Mechanism}

The data prioritization and delay modeling are performed by the application layer by considering the MAC layer parameters according to the requirements of industrial applications and the network conditions. The IEEE 802.15.4 standard uses Carrier Sense Multiple Access with Collision Avoidance (CSMA/CA) to access the radio channel. However, CSMA/CA is not suitable for the delay critical industrial applications since it does not include the prioritization and delay responsiveness properties [19]. In the industrial IoT systems, flow control, process monitoring, and fault detection sub-system must have priority and delay aware medium access mechanisms.

Figure 2 shows the timing diagram of different nodes contending the channel access according to their priority levels. In this scenario, any packets in the low priority queue will not be served until the high priority queue becomes empty. The $P_{h}$ nodes always have the fixed short backoff period, more frequent Common Channel Access (CCA) detection, and high number of backoffs. However, the $P_{l}$ nodes use longer random backoff period, less frequent detection, and lower number of backoffs. In addition, CCA detection time of $P_{l}$ nodes is considered to be longer than the sum of CCA detection time and the backoff periods of $P_{h}$ nodes.

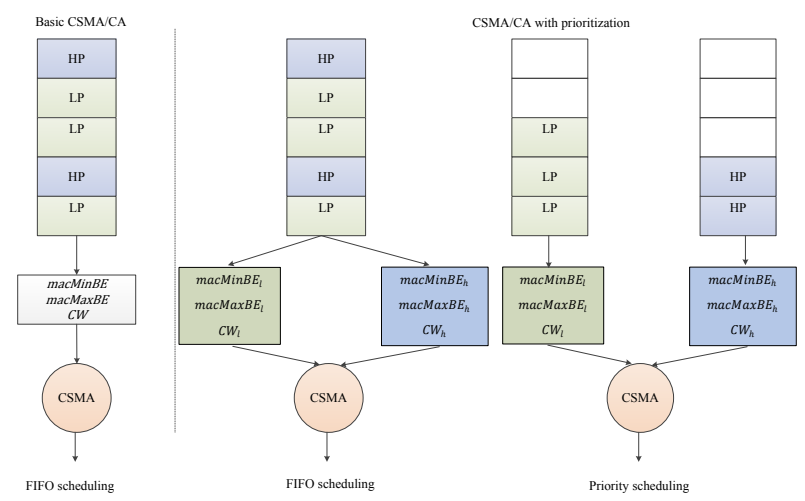

Fig. 3. Illustrations of the basic CSMA/CA as First-In-First-Out (FIFO) scheduling and the prioritized CSMA/CA.

The behavior of the CSMA/CA is affected by different MAC parameters such as the minimum backoff exponent ( macMinBE), the maximum backoff exponent (macMaxBE), the intial value of the contention window $(C W)$, and the maximum number of backoffs (macMaxCSMABackoffs). Different values of these MAC parameters have a great impact on the performance of an IoT network. Instead of having the same value of CSMA/CA parameters for both traffic (i.e., low priority and high priority), we can assign its own attributes for each class. Let us define $\left[\operatorname{macMinB} E_{h}, \operatorname{macMaxB} E_{h}\right]$ and $C W_{h}$ as the backkoff interval and contention window values for

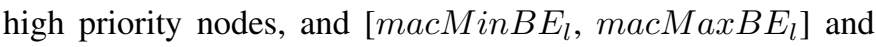
$C W_{l}$ as the corresponding values for the low priority nodes. Moreover, by specifying different CSMA/CA parameters, the priority based scheduling can be implemented to reduce the channel access latency of the high priority packets as depicted in Fig. 3 [19].

\section{B. Data Aggregation without Prioritization}

In case of data aggregation without prioritization, the data packets from sensor nodes arrive at the $\mathrm{CH}$ and are placed on the queue. The individual packets at the $\mathrm{CH}$ are served in different time lengths. In the considered M/G/1 queuing model, the data arrival pattern follows the Poisson distribution with a packet arrival rate $\lambda$, and the utilization rate of the packet at the $\mathrm{CH}$ is given by

$$
\rho=\lambda E[S],
$$

where $E[S]$ is the expected service time of the aggregated data without priority. The expected waiting time $E[W]$ of the nonpriority agrregated data before being served and the expected system delay $E\left[D_{\text {sys }}\right]$, i.e., the total time that the packet should be in the queue until being transmitted as an aggregated data can be expressed as [11]

$$
\begin{gathered}
E[W]=\frac{\rho E\left[S^{2}\right]}{2(1-\rho)}, \\
E\left[D_{\text {sys }}\right]=E[S]+E[W],
\end{gathered}
$$

where $E\left[S^{2}\right]$ is the second order moment of the service time, and can be computed as follows

$$
E\left[S^{2}\right]=\frac{4}{3} E[S]^{2} .
$$


In this paper, we adapt the general mathematical model of CSMA/CA procedure of IEEE 802.15.4 presented in [17,20]. Using this model, the expected service time can be expressed as [17]

$$
E[S]=E[D]+T_{T x}+2 T_{t u r n}+T_{A C K},
$$

where $E[D]$ denotes the time duration from the epoch that the data packet just arrives at the head of queue to the epoch just before packet transmission or discarded. The $T_{T x}$ and $T_{A C K}$ are the transmission time of data and acknowledgment packet respectively, and $T_{t u r n}$ is the turnaround time. The parameter $E[D]$ depends on the CSMA/CA procedure and is affected by different MAC parametes such as $C W, \operatorname{macMax} B E$, macMinBE, and macMaxCSMABackoffs. The expected value $E[D]$ can be expressed as [17]

$$
\begin{aligned}
E[D]= & \sum_{v=0}^{m} \alpha^{v}(1-\alpha)\left\{\sum_{i=0}^{v} \frac{C W_{i}-1}{2} \sigma+(v+1) T_{C C A}\right\} \\
& +\alpha^{m+1}\left\{\sum_{i=0}^{m} \frac{C W_{i}-1}{2} \sigma+(m+1) T_{C C A}\right\},
\end{aligned}
$$

where $T_{C C A}$ is the time interval for performing CCA, $\alpha$ is the busy channel probability, and $\sigma$ is the length of backoff slot. The contention window size for the $i$ th retry is given by; $C W_{i}=\min \left\{2^{i} \operatorname{macMinBE}, \operatorname{macMax} B E\right\}$. The default values of macMinBE and $\operatorname{macMaxBE}$ are 3 and 5, respectively [17]. The data packets are discarded or dropped after $m+1$ attempts at CCA, and subsequently the data packet loss rate is given by [17]

$$
P_{\text {loss }}=\alpha^{m+1} .
$$

Then, the probability of channel being busy $\alpha$ can be expressed in term of $P_{\text {loss }}$ as [17]

$\alpha=\frac{(N-1)\left(1-P_{\text {loss }}\right) E\left[N_{\tau}\right]\left(T_{C C A}+T_{T x}+2 T_{\text {turn }}+T_{A C K}\right)}{\frac{1}{\lambda}+E\left[N_{\tau}\right] E[D]}$,

where $N$ is the number of sensor nodes associated with the $\mathrm{CH}, N_{\tau}$ is the number of packets served in a busy period of the $\mathrm{M} / \mathrm{G} / 1$ queuing system, and $E\left[N_{\tau}\right]=\frac{1}{1-\rho}$. Therefore, by solving the non-linear equations (7), (8), and (9), we can obtain the corresponding values of $\alpha, P_{\text {loss }}$, and $E[D]$.

\section{Data Aggregation with Prioritization}

In the case of data aggregation with prioritization, the prioritized $\mathrm{M} / \mathrm{G} / 1$ queuing model holds $P$ priority class of data. The packets with the $i$ th priority have arrival rate $\lambda_{i}$, $i \in\{1,2, \ldots, P\}$, and follow the Poisson distribution. The lower value of $i$ indicates a high priority packet type. In our system model, we implement a preemptive priority rule, i.e., the new arrival of class $i$ th priority packet will immediately preempt lower priority data currently being served and get the access to the services. The workflow diagram of the proposed scheme is presented in Fig. 4.

The waiting time $W_{i}$ of the $i$ th priority packet is the time spent in the queue before being served at the $\mathrm{CH}$. The mean residual service time for the packets currently being served and the service time of the $\mathrm{CH}$ are denoted by $R_{i}$ and $S_{i}$, respectively. The overall system delay is given by the summation of the waiting time and the service time of the packets. By using the Little's law, the expected waiting time of the $i$ th priority packet is given by

$$
E\left[W_{i}\right]=\frac{\sum_{j=1}^{i} \rho_{j} E\left[R_{j}\right]}{\left(1-\left(\rho_{1}+\ldots+\rho_{i}\right)\right)\left(1-\left(\rho_{1}+\ldots+\rho_{i-1}\right)\right)},
$$

where $\rho_{i}=\lambda_{i} E\left[S_{i}\right], E\left[S_{i}\right]$ is the expected service time, and $E\left[R_{i}\right]$ represents the expected residual time. Let $E\left[\hat{S}_{i}\right]$ and $E\left[\hat{D}_{i}^{s y s}\right]$ are the expected service time of $i$ th priority packet by considering the interruptions of higher priority packet and the expected system delay in the $i$ th priority queue respectively, and are calculated by

$$
\begin{gathered}
E\left[\hat{S}_{i}\right]=\frac{E\left[S_{i}\right]}{\left(1-\left(\rho_{1}+\ldots+\rho_{i-1}\right)\right)}, \\
E\left[\hat{D}_{i}^{s y s}\right]=E\left[\hat{S}_{i}\right]+E\left[W_{i}\right] .
\end{gathered}
$$

In addition, the service time $E\left[S_{i}\right]$ of the $\mathrm{CH}$, the expected residual time $E\left[R_{i}\right]$, and the second-order moment of the service time $E\left[S_{i}^{2}\right]$ for the priority-based data aggregation can be expressed as [13]

$$
\begin{gathered}
E\left[R_{i}\right]=\frac{2}{3} \lambda_{i} E\left[S_{i}\right]^{2} . \\
E\left[S_{i}^{2}\right]=\frac{4}{3} E\left[S_{i}\right]^{2},
\end{gathered}
$$

Similarly, the value of $E\left[S_{i}\right]$ can be calculated by using (6), (7), (8), and (9) in accordance with the values of $C W_{i}$ and $\lambda_{i}$.

\section{Performance Analysis}

In this section, we evaluate and analyze the performance of the proposed scheme in terms of the expected system delay and system reliability. The simulation parameters are listed in Table I [21]. We used MATLAB software in order to obtain the results presented in this paper.

TABLE I

Simulation PARAMENTERS

\begin{tabular}{cc}
\hline Parameters & Value \\
\hline Max Backoff Exponent & 5 \\
Min Backoff Exponent & 3 \\
Max CSMA Backoff & 4 \\
MAC Frame Payload & 800 bits \\
Queue Size & 51 frames \\
Data Rate & $19.2 \mathrm{kbps}$ \\
ACK Size & $88 \mathrm{bits}$ \\
MAC Overhead & $48 \mathrm{bits}$ \\
$\sigma$ & $0.32 \mathrm{~ms}$ \\
$T_{A C K}$ & $0.352 \mathrm{~ms}$ \\
$T_{T x}$ & $1.12 \mathrm{~ms}$ \\
$T_{C C A}$ & $0.25 \mathrm{~ms}$ \\
$T_{t u r n}$ & $0.192 \mathrm{~ms}$ \\
\hline
\end{tabular}




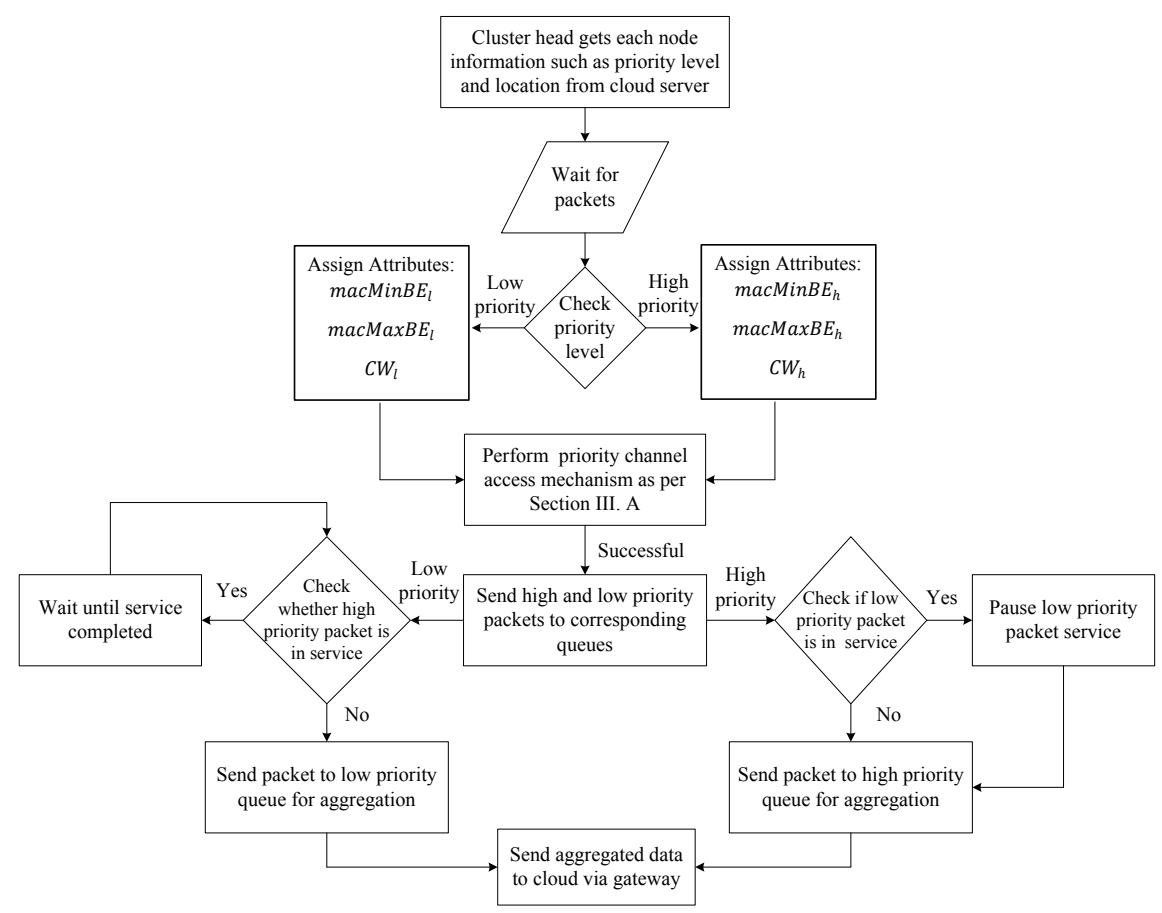

Fig. 4. Workflow diagram of the proposed scheme.

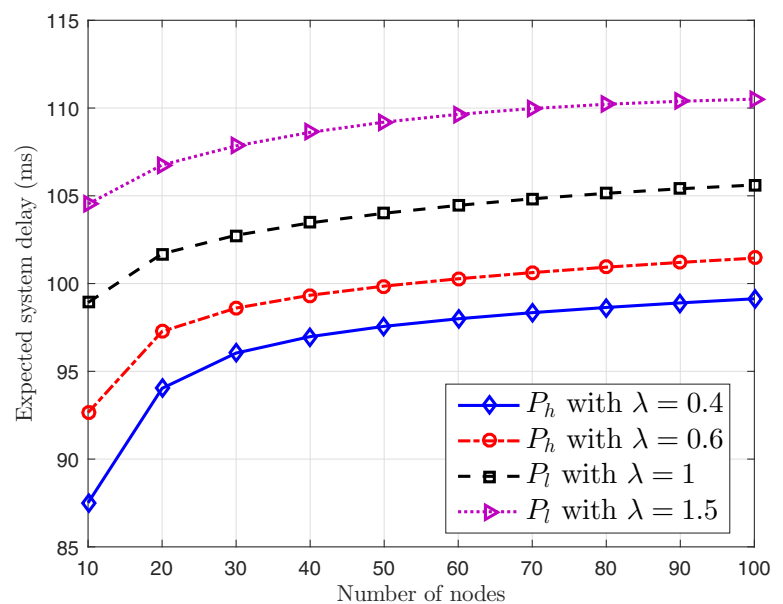

Fig. 5. Performance evaluation of the proposed priority approach in terms of the expected system delay.

\section{A. Expected System Delay}

Figure 5 presents the expected system delay of packets with different priority levels versus the number of sensor nodes. The expected system delay of both high and low priority packets increases as the number of node increases because aggregation of higher number of data packets yields the longer service time. The low priority packets have the longer delay as compared to that of the high priority packets because the service time must accommodate the interruptions of all packets with the higher priority. Similarly, Fig. 6 shows the performance comparison of the proposed priority scheme with the non-priority scheme. The non-priority scheme has a similar characteristic curve; however, the delay is higher than the priority scheduling approach. Moreover, due to the prioritized

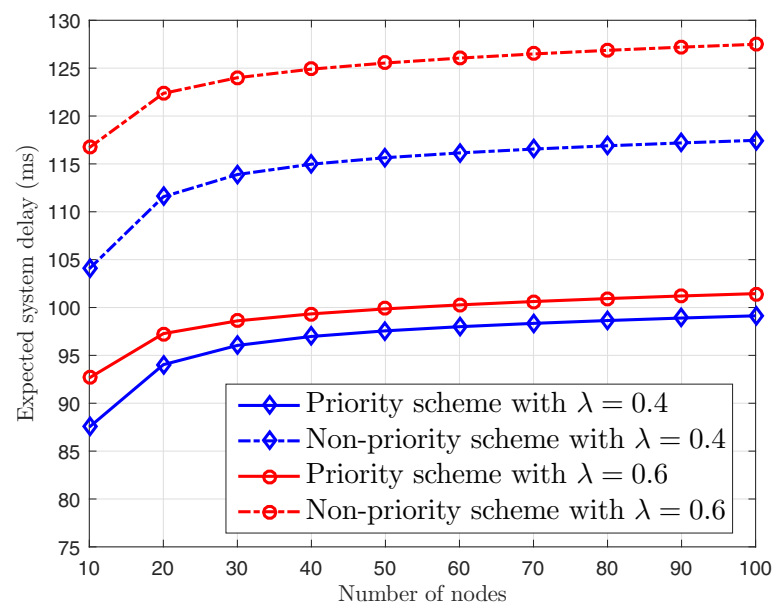

Fig. 6. Performance comparison of the proposed priority approach with nonpriority scheme in terms of the expected system delay.

channel access mechanism and preemptive priority rule, the high priority packets do not get any interruptions from the low priority packets and hence, the expected system delay is reduced.

\section{B. Reliability}

The proposed scheme is modeled as the preemptive M/G/1 priority queue with the system size $K$ and each queue receives data frames by following the Poisson arrival process with the rate of $\lambda$ data packets per second. The steady state probability that $i$ data packets are present in the queue is given by [21]

$$
p_{i}=\frac{\rho^{i}}{\sum_{j=0}^{K} \rho^{j}} .
$$




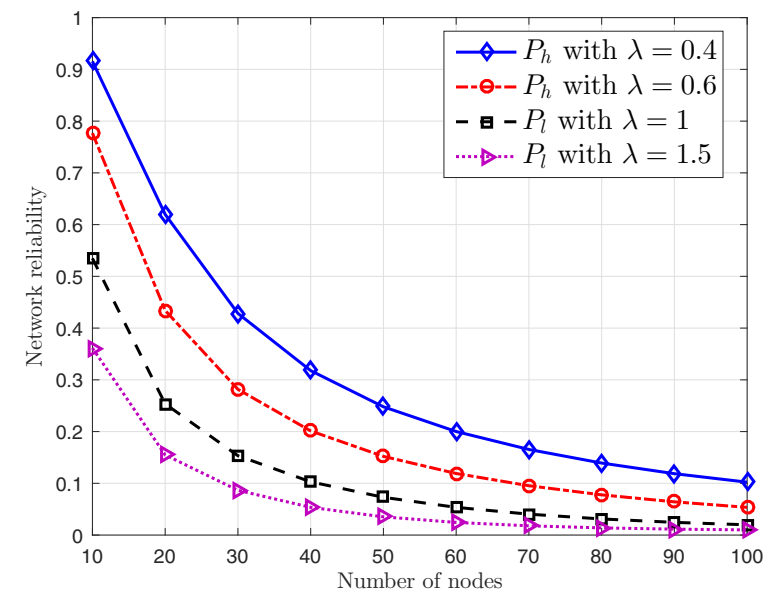

Fig. 7. Performance evaluation of the proposed priority approach in terms of network reliability.

The different possibilities that sensor nodes may not be able to successfully send data packets to the $\mathrm{CH}$ include: (i) if the buffer is full, (ii) if nodes fail to find the idle channel, and (iii) the packets are discarded after exceeding retry limits. By considering these aspects, the system reliability $\eta$ can be calculated as [21]

$$
\eta=\left(1-p_{k}\right)\left(1-P_{c f}\right)\left(1-P_{c r}\right),
$$

where $p_{k}$ is the probability of having full buffer with $k$ frames and is given by (15), $P_{c f}$ is the probability that the packet is dropped due to the channel access failure, and $P_{c r}$ is the probability of packet discarded due to the retry limit.

Figure 7 depicts the overall system reliability versus the number of nodes. It is clearly observed that the network reliability decreases as the number of nodes increases. Each node in the queue begins to experience the congestion problems due to a large number of nodes; collisions become more frequent, and the packet re-transmissions are more recurrent. Subsequently, the delays get longer as the queues become busier. The probability of frame loss also increases because of the collisions, the retry limits, and the link constraints. Moreover, due to the employed priority-based channel scheduling mechanism and queuing policy, the network reliability of the high priority nodes is noted to be higher than that of the low priority nodes.

\section{Conclusions}

IoT networks consist of a large number of sensor nodes for different sensing and monitoring purposes. The resourceconstrained IoT devices operating in highly dense networks may be affected by the data collisions, packet loss, packet delays and low network throughput. These IoT devices usually have diverse data traffic with different latency and system reliability requirements. In this paper, we proposed a cloudassisted latency minimization scheme by using prioritized channel access and data aggregation at the $\mathrm{CH}$. In addition, we considered the joint impact of packet scheduling and aggregation by using the preemptive M/G/1 queuing model. With the help of numerical results, it has been shown that the prioritized channel access and data aggregation scheme provides substantial improvements in terms of latency and system reliability as compared to the non-prioritized scheme. In our future work, we plan to use network simulator tools to analyze the performance of the proposed scheme in realworld IoT applications such as eHealthcare and industrial automation.

\section{REFERENCES}

[1] L. Atzori, A. Iera, and G. Morabito, "The Internet of Things: A survey," Comput. Net., vol. 54, no. 15, pp. 2787-2805, 2010.

[2] M. Jutila, "An adaptive edge router enabling Internet of Things," IEEE Internet Things J., vol. 3, no. 6, pp. 1061-1069, Dec 2016.

[3] S. Bhandari, S. K. Sharma, and X. Wang, "Cloud-assisted device clustering for lifetime prolongation in wireless IoT networks," in Proc. IEEE CCECE, April 2017, pp. 1-4.

[4] L. D. Xu, W. He, and S. Li, "Internet of Things in industries: A survey," IEEE Trans. Ind. Informat., vol. 10, no. 4, pp. 2233-2243, Nov 2014.

[5] S. K. Sharma, T. E. Bogale, S. Chatzinotas, X. Wang, and L. B. Le, "Physical layer aspects of wireless IoT," in Proc. IEEE ISWCS, Sept 2016, pp. 304-308.

[6] N. Nasser, L. Karim, and T. Taleb, "Dynamic multilevel priority packet scheduling scheme for wireless sensor network," IEEE Trans. Wireless Commun., vol. 12, no. 4, pp. 1448-1459, April 2013.

[7] IEEE 802.15.4, "Wireless Medium Access Control (MAC) and Physical Layer (PHY) specifications for Low Rate Wireless Personal Area Networks (WPANs)," IEEE Std 802.15.4-2006, pp. 1-320, Sept 2006.

[8] I. Park, D. Kim, and D. Har, "MAC achieving low latency and energy efficiency in hierarchical M2M networks with clustered nodes," IEEE Sensors J., vol. 15, no. 3, pp. 1657-1661, March 2015.

[9] H. Yan, Y. Zhang, Z. Pang, and L. D. Xu, "Superframe planning and access latency of slotted MAC for industrial WSN in IoT environment," IEEE Trans. Ind. Informat, vol. 10, no. 2, pp. 1242-1251, May 2014.

[10] S. Jardosh and P. Ranjan, "EPCAP: Explicit Prioritized Channel Access Protocol for IEEE 802.15.4 based wireless sensor networks," in Proc. IEEE WCNC, April 2013, pp. 1-6.

[11] N. Kouzayha, M. Jaber, and Z. Dawy, "M2M data aggregation over cellular networks: signaling-delay trade-offs," in Proc. IEEE Globecom Wkshps, Dec 2014, pp. 1155-1160.

[12] G. Rigazzi, N. K. Pratas, P. Popovski, and R. Fantacci, "Aggregation and trunking of M2M traffic via D2D connections," in Proc. IEEE ICC, June 2015, pp. 2973-2978.

[13] S. A. AlQahtani, "Analysis and modelling of power consumption-aware priority-based scheduling for M2M data aggregation over long-termevolution networks," IET Commun., vol. 11, pp. 177-184(7), Jan 2017.

[14] M. M. Hassan, H. S. Albakr, and H. Al-Dossari, "A cloud-assisted internet of things framework for pervasive healthcare in smart city environment," in Proc. EMASC. ACM, 2014, pp. 9-13.

[15] S. K. Sharma and X. Wang, "Live data analytics with collaborative edge and cloud processing in wireless IoT networks," IEEE Access, vol. 5, pp. 4621-4635, 2017.

[16] S. Mubeen, P. Nikolaidis, A. Didic, H. Pei-Breivold, K. Sandstrom, and M. Behnam, "Delay mitigation in offloaded cloud controllers in industrial IoT," IEEE Access, vol. 5, pp. 4418-4430, 2017.

[17] T. O. Kim, J. S. Park, H. J. Chong, K. J. Kim, and B. D. Choi, "Performance analysis of IEEE 802.15.4 non-beacon mode with the unslotted CSMA/CA," IEEE Commun. Lett., vol. 12, no. 4, pp. 238240, April 2008.

[18] M. Doudou, D. Djenouri, and N. Badache, "Survey on latency issues of asynchronous MAC protocols in delay-sensitive wireless sensor networks," IEEE Commun. Surveys Tuts., vol. 15, no. 2, pp. 528-550, Second 2013.

[19] A. Koubaa, M. Alves, B. Nefzi, and Y.-Q. Song, "Improving the IEEE 802.15.4 Slotted CSMA/CA MAC for Time-Critical Events in Wireless Sensor Networks," in Proc. RTN, ECRTS Wkshps, July 2006.

[20] P. Park, P. D. Marco, P. Soldati, C. Fischione, and K. H. Johansson, "A generalized markov chain model for effective analysis of slotted IEEE 802.15.4," in Proc. IEEE MASS, Oct 2009, pp. 130-139.

[21] M. H. Zayani, V. Gauthier, and D. Zeghlache, "A joint model for IEEE 802.15.4 physical and medium access control layers," in Proc. IWCMC, July 2011, pp. 814-819. 